\title{
Impact of radio randomness on performances of lattice wireless sensors networks based on event-reliability concept
}

\author{
T. Yang, G. De Marco*, M. Ikeda and L. Barolli \\ Department of Information and Communication Engineering, Fukuoka Institute of Technology (FIT), \\ 3-30-1 Wajiro-Higashi-ku, Fukuoka 811-0295, Japan
}

\begin{abstract}
The problem of congestion control in wireless sensor networks is very important, especially in very dense networks. In this work, we use a simple packet repetition scheme as congestion and event-reliability control. In contrast to other works on the performance study of such a scheme, we use a random radio pathloss model. We are motivated by this choice, because the radio model usually used in most of studies on wireless sensor networks is deterministic. However, this is not the case encountered in real hardware. Here, we show that the analysis of such a scheme by simulation is not trivial when more realistic radio models are used. We emphasise this problem by comparing the differences of simulation results with respect to the non random pathloss case, also known as isotropic model. As expected, simulations confirm that radio irregularities can not be neglected and the efficiency of event-reliability based congestion control should be properly revised. This aspect calls for new mechanisms of coordination among sensor nodes.
\end{abstract}

Keywords: Wireless sensor networks, congestion control, sensor nets simulation

\section{Introduction}

A Wireless Sensor Network (WSN) is a wireless network where the nodes are sensors, that is microdevices with limited computation capacity and with on-board specific transducers. These kind of networks can facilitate the monitoring of the environment and hazardous area [16]. Although the number of nodes depends on the application and many other system factors, it is recognised that typical applications of WSNs will contain thousand of sensor nodes. The performance analysis of a large scale WSN is possible only by means of simulation. In fact, the design and on-field test approach is too expensive for the analysis of protocols and applications of typical WSNs. Accordingly, often researchers study the basic properties of WSN by means of general purpose event-driven simulators, as the well known Network Simulator Version 2 (NS-2). There are also other ad hoc simulators. For example, the Emstar [8] and the TOSSIM [11] simulators are well engineered simulators for WSNs. However, when the aim of the simulation is not the implementation of the simulation code into real sensor nodes, a general purpose simulator fits the aims. Besides many other details of the dynamics of multi-hop communications in WSN, one of them which has quite neglected so far is the model of the physical environment implemented in the simulators. This model requires the details of the monitored

\footnotetext{
${ }^{*}$ Corresponding author. Tel.: +8192 606 4970; E-mail: \{demarco, barolli\}@ fit.ac.jp.
} 
phenomenon, its space-time characteristics, and the details of the radio medium. In most studies on WSNs and wireless networks as well, the radio model usually used is spherical or isotropic. This means that the received power at a certain distance is the same in all directions. Every radio engineer knows that this is not the case encountered in real hardware, i.e. in the real network the received power level fluctuates in the space and in the time because of the heterogeneity of the surrounding environment. Although the right model depends mainly on the type of environment (e.g. the signal propagation in a forest is different than in a city), recently, the importance of considering more accurate models has been shown to be a key factor on establishing the performance of MAC and routing protocols in ad hoc and sensor networks $[4,19,20]$. In particular, reactive routing protocols as the Ad hoc On Demand distance Vector (AODV) and Dynamic Source Routing (DSR) [13] can well react against the irregularities of the radio medium by means of multiple round path discovery and multiple paths [20], at the cost of increased routing overhead. On the contrary, proactive routing protocols as Optimized Link State Routing (OLSR) [5] maintain a routing table for every destination inside the network. Moreover, in the case of OLSR advanced mechanisms such as monitoring of link quality and clustered flooding permits to ameliorate the connectivity and the average packet latency of the network. However, proactive protocols are too expensive for energy contrained sensor nodes, because a periodic update of the routing tables is necessary. Other routing protocols, such as Geographical Forwarding (GF) makes use of the geographical position of nodes in order to compute the communication path. Such protocols suffer much radio irregularities than other ones, because the geographic closeness of a node does not imply its radio reachability. In this work, we focus our review on the dependence of simulation results on the model of the radio medium. We emphasise this problem by comparing the impact of realistic simulations on the study of event-reliability based congestion control. As expected, simulations confirm that radio irregularities can not be neglected. Besides the review of current studies on performance analysis, we give some basic simulations carried out by means of NS-2 and public available libraries. Our findings concern the fact that, as expected, simplistic models can be harmful to the evaluation of routing protocols and WSN applications. Furthermore, since WSNs can be composed by hundred of micro-sensors, the design of simulation experiments is not trivial. For example, the simulation time depends much on the desired accuracy of results. Accordingly, more advanced simulation techniques are required in order to take into account wireless channel models and to bound the simulation time as well.

\section{Simulation of sensor nodes}

Before entering into the main discussion of the work, let us explain the method we used in order to simulate a WSN. We used the NS-2 [10] along with the libraries of Naval Research Laboratory (NRL) [7]. These libraries model the generic physical event (temperature, pressure, sound, heat) as a mobile node which emits at a constant rate a packet of fixed size. This packet is then broadcasted over the radio medium with a certain power, by assuming that the propagation laws of the physical event are the same as the electromagnetic signal propagation. In fact, by looking at the Fig. 1, we note that the event node has the same physical and network layer of the sensor node network stack. In NS-2, we can choose two types of radio model: deterministic and random. For the deterministic model, the range of the emitted signal is constant along all directions, i.e. the coverage area is a perfect circle centred at the node position. ${ }^{1} \mathrm{We}$ will use this deterministic and isotropic assumption for the propagation of the natural event, although the

\footnotetext{
${ }^{1}$ If the antenna is omni-directional.
} 


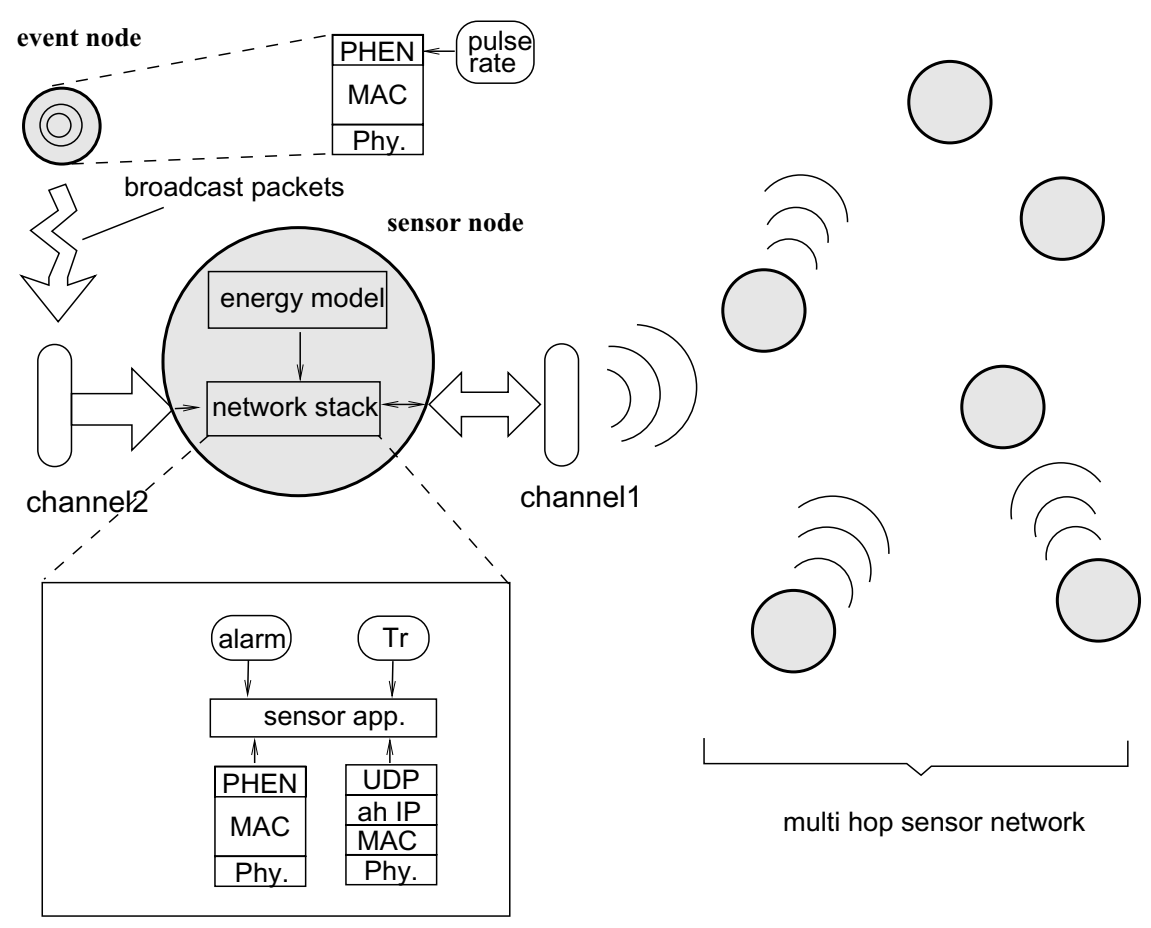

Fig. 1. Sensor model.

real propagation mechanisms can be different. In general, every signal can be detected at the physical layer if its received power is greater then a threshold, $\mathrm{RX}_{\mathrm{tr}}$. In Fig. 1, we have shown also the model of the sensor. The sensor node has two channels. In the NS-2 terminology, these channels are two distinct instances of the class representing the radio medium. One channel (channel 1) is dedicated to the communication with other neighbouring nodes, and nodes use specific routing and MAC protocols. The second channel (channel 2) is used to model the sensing operations. On this channel, the sensor node hears broadcast packets from the event node. A special routing agent called PHENOM is used. The event node has two parameters, that is the pulse rate and the radiated power. On the sensor side, we can set a lot parameters. For example, we can set the sensing range of the sensor by changing the threshold at the physical layer of the channel used for receiving packets of the event node. We can also set an alarm variable, which is the time during which the sensor remains active. If the alarm timeouts, the sensor goes into an inactive zone, and no more (data) packets are being sent. The sensor node can use a number of routing and transport protocols as well. However, we will use the UDP protocol.

\section{The radio model}

There are two basic models for the propagation of the radio signals of sensor nodes. The first one is a simple deterministic model, where the received power $P_{r}$ at a certain distance $d$ is the same along all directions in the plane. ${ }^{2}$ For example, in the case of Line Of Sight (LOS) propagation of the signal, the

\footnotetext{
${ }^{2}$ We are considering 2D networks, but similar results hold also in the more general case of tridimensional networks.
} 
Friis formula predicts the received power as:

$$
\begin{aligned}
& P_{r}(d)=P_{t}-\beta(\mathrm{dB}), \\
& \beta=10 \log \left(\frac{(4 \pi d)^{2} L}{G_{t} G_{r} \lambda^{2}}\right)
\end{aligned}
$$

where $G_{r}$ and $G_{t}$ are the antenna gains of the receiver and the transmitter, respectively, $\lambda$ is the wavelength of the signal and $L$ the insertion loss caused by feeding circuitry of the antenna, and $\beta$ is the propagation pathloss. For omni-antennas, $G_{R}=G_{t}=1$. The signal decay is then proportional to $d^{2}$. A more accurate model is the so called Two-Ray-Ground model, where in addition to the direct ray from the transmitter towards the receiver node, a ground reflected signal is supposed to be present. Accordingly, the received power now depends also on the antenna heights and the pathloss is:

$$
\beta=10 \log \left(\frac{(4 \pi d)^{4} L}{G_{t} G_{r} h_{t} h_{r} \lambda^{2}}\right)
$$

where $h_{r}$ and $h_{t}$ are the receiver and transmitter antenna heights, respectively. The power decreases faster than Eq. (1). The formula in Eq. (2) is valid for distances $d>d_{c}$, that is far from the transmitting node. A more general model is the Shadowing model. In this model, the pathloss is divided in two parts, one being deterministic and the other being a random variable (r.v.). By introducing a reference distance $d_{0}$, in decibels, we have:

$$
\begin{array}{r}
\beta(d)=\beta_{0}+X_{\sigma} \\
\overline{P_{r}(d)}=P_{r}\left(d_{0}\right)-\beta_{0}=P_{r}\left(d_{0}\right)-10 \alpha \log \left(d / d_{0}\right),
\end{array}
$$

where $\alpha$ is a generic pathloss exponent which depends on the type of environment [14]. The value in Eq. (4) is the mean value of the received power, and the actual received power is $P_{r}(d)=\overline{P_{r}(d)}+X_{\sigma}$, where $X_{\sigma}$ is a r.v. with a zero mean Gaussian distribution and variance $\sigma^{2}$. Note that the pathloss is then log-normal distributed. We can see that the variations of the pathloss are non-uniform along all directions. The range $r_{0}$ of the emitted signal is defined as the maximum distance after which the signal cannot be detected. In other words, the pathloss $\beta$ should be lower than a pathloss threshold, $\beta_{\mathrm{th}}$. This value obviously depends on the sensitivity of the receiver, $R X_{t r}$. Accordingly, we have:

$$
r_{0}=10^{\frac{\beta_{\mathrm{th}}-X}{10 \alpha}} .
$$

The presence of $X_{\sigma}$ makes also $r_{0}$ a r.v. Because of the log-normality of the pathloss, the magnitude of the range variations can be very high. For example, for $\beta_{\text {th }}=40 \mathrm{~dB}$, the transmission range can be as high as $200 \mathrm{~m}$, i.e. double times the range in the case of deterministic model, as shown also in Fig. 2. We can see that the overall effect of the Shadowing is a non negligible probability that nodes near to the transmitter do not hear its signal, and there is also a non negligible probability of nodes very far that can hear the transmitter's signal. We will see in the next Sections that the non circularity of the radio range impacts the performances of the WSN. There are also other more accurate models. The Shadowing model is also called large-scale variations fading, and it is due to non-uniform properties of surrounding clutters. Its counterpart is the small-scale fading or simply fading, which takes into account the rapid variations of the pathloss due to random movements of surrounding objects, the multi-path propagation of the signal and, eventually, the relative movement of the transmitter and receiver nodes. 
The distribution of the signal strength usually adopted in the case of fading is the Rice distribution. ${ }^{3}$ The authors in [19], proposed a Weibull distribution as a generalisation of the Rayleigh distribution in order to model the data of their WSN test-bed. On the other hand, authors in [12] suggest that a Two-Ray-Ground model is enough for outdoor ground-mounted WSNs.

The right model to use clearly depends on the particular environment of deployment of the WSN. Here, we are not concerned with the right choice of the radio model, but rather we are interested in the impact of the radio model on the algorithms commonly used for WSNs. In particular, two aspects are surely important. First, the routing layer of sensor nodes behaves in a different way if we suppose anisotropic radio range. Secondly, the applications running on top of the sensor nodes will suffer much or less the randomness of the pathloss. We will discuss these two aspects in the following Sections.

\section{The impact on routing protocols}

To the best knowledge of the authors, only the work in [20] addresses the interaction of the radio irregularities and the performances of both MAC and routing protocols. In particular, the authors show that the irregularities have a relatively larger impact on the routing layer than the MAC layer. Furthermore, in that work the dependence of the signal anisotropy on the hardware heterogeneity, i.e. variations of battery energy, is also argued out. Let us show some examples of the effects of these impairments. In general, we have two types of routing protocols for ad hoc networks: reactive and proactive protocols. Reactive protocols build up a route whenever a data packet is to be transmitted. Consequently, a route discovery procedure precedes the transmission of data packet. On the other hand, proactive protocols maintain a routing table inside the nodes. The routing tables are periodically updated by means of "HELLO" packets. Since reactive protocols do not consume energy to maintain updated routing tables, proactive or on demand protocols are often preferred in WSNs. However, this benefit has a cost on the mean latency of transmission. Examples of reactive protocols are the well known AODV and the DSR [13]; proactive protocols are the Destination-Sequenced Distance-Vector (DSDV) [13] and the OLSR [5]. OLSR is currently under revision by the Internet Engineering Task Force (IETF) in view of a standardisation as ad hoc routing protocol. As all proactive protocol, OLSR can discover the state of neighbouring links, whether they are symmetric or not. Moreover, it can reduce broadcast traffic by means of multipoint relaying concept, which resembles standard clustering in ad hoc network. However, the trade-off energy/delay remains. Here, we consider reactive routing protocols only.

When a data packet must be transmitted from a source node to a destination node, these protocols first broadcast a Route REQuest (RREQ) message. Every node which receives this message increases the hop counter inside the packet, and, if it is not the destination or the node which sent the packet, re-broadcasts the packet. By this way, if the network is connected, the destination will eventually be found. When the destination node receives the RREQ packets, it sends back to the source a Route REPly packet (RREP). The destination can choose a reverse path from its own cached routes, or, in the case of a cache miss, initiates a discovery procedure for the reverse path. The inherent assumption which has been considered so far is that if a path from a source to destination exists, also the reverse path from the destination to the source exists. By this assumption, the destination uses the reversed sequence of hop appearing in the header of the RREQ message. For instance, this is the procedure pursued by the DSR protocol. In AODV, there is also a special route maintenance mechanism, by which every node can easily

\footnotetext{
${ }^{3}$ Under the assumption that LOS ray is present.
} 

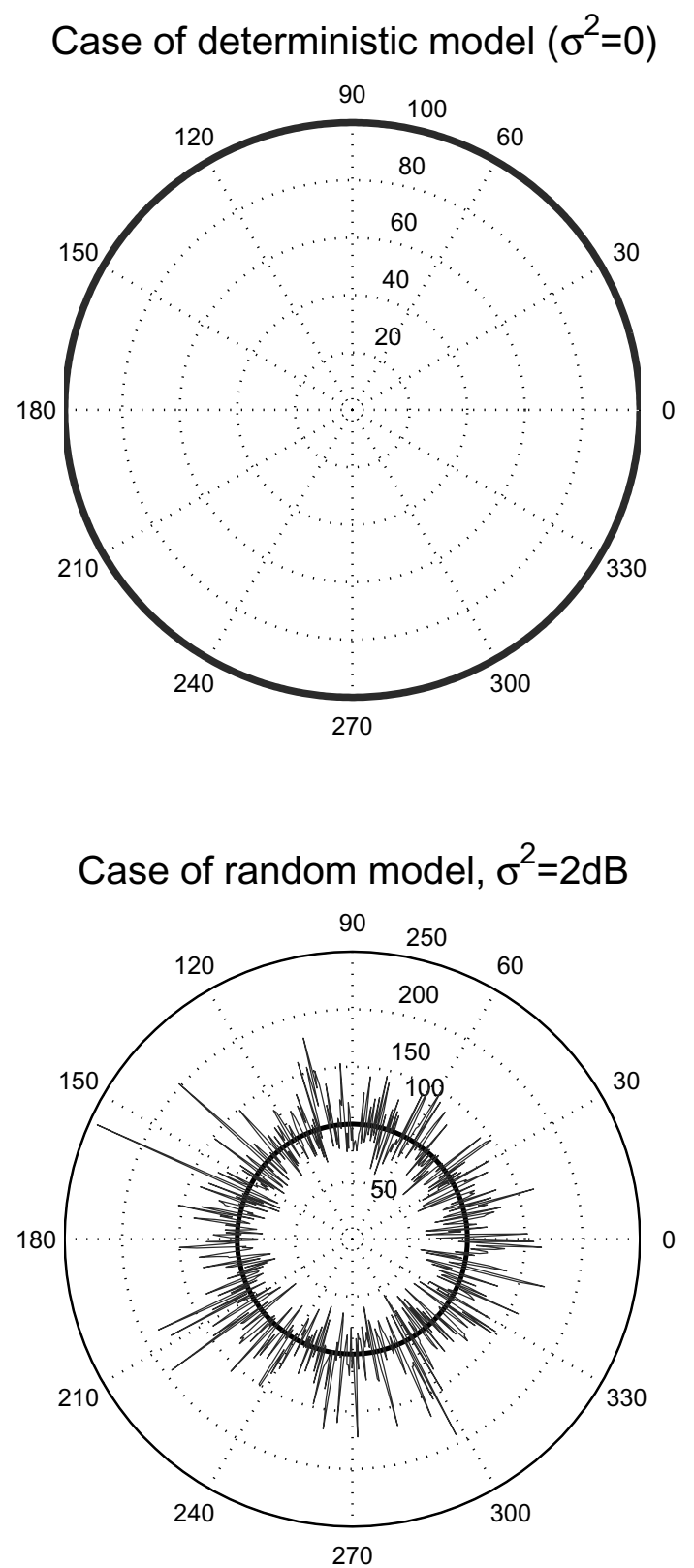

Fig. 2. Transmission range with respect to two radio models. Pathloss threshold is $\beta_{\mathrm{th}}=40 \mathrm{~dB}$.

knows the state of the links to its neighbours. This mechanism is based on broadcast messages called "HELLO" messages, which periodically request a feedback from neighbouring nodes. Upon receiving these messages, the neighbouring nodes in turn send back a message of the sort "I AM ALIVE" in order to keep updated the routing table of the requesting node. If a link is inactive for some reason, the routing table will be adequately updated. This mechanism is very useful against node and/or link failures, but it incurs in some extra energy consumption. Moreover, the setting of the "HELLO" messages rate is not a trivial problem, especially in very dense networks where broadcast transmissions can lead to a congestion 


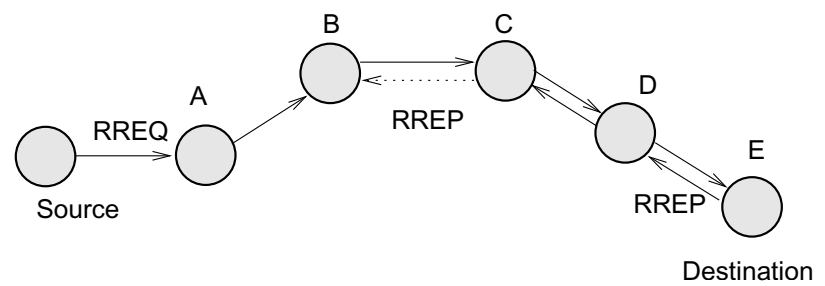

(a) Single-round discovery

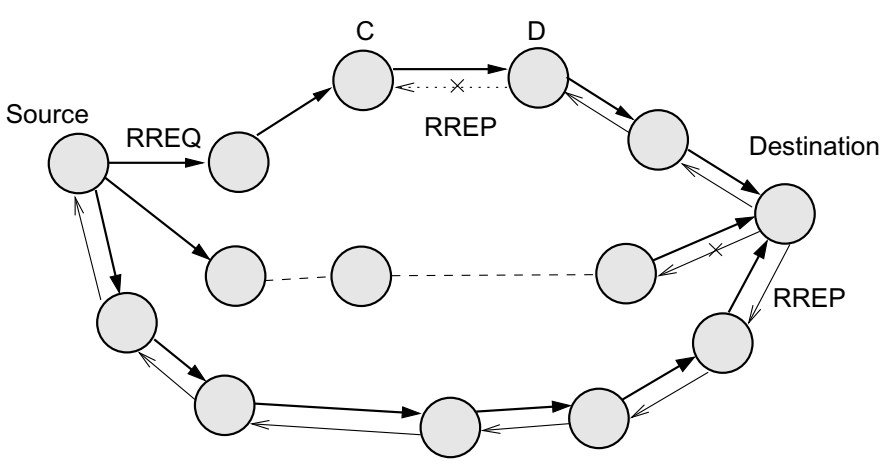

(b) Multi-round discovery concept

Fig. 3. Effects of link asymmetries on route discovery of reactive protocols.

collapse. However, from the discussion in the Section 3, we know that the assumption of symmetric path is not the case encountered in the real hardware. As shown also in Fig. 3(a), although the route discovery procedure discovered the path $\mathrm{ABCDE}$, the destination cannot use the reverse route, that is DCBA, because the link CD is not symmetric. Moreover, a link previously marked as "active" or rather as the presence of a neighbouring node could not be a neighbour at all in the successive transmission instances. From a theoretical point of view, the availability of a bi-directional path depends on the connectivity properties of the networks. The connectivity of the network in turn strongly depends on the radio channel parameters and the density of the network. By far, theoretic results on the connectivity of ad hoc networks have been obtained with the assumption of symmetric links $[13,14]$, and they could not apply to real scenarios where the links asymmetry is all but a negligible property.

However, in general, on demand protocols make use of the so called multi-rounds route discovery procedure which can minimise the impact of the radio irregularities. Basically, this technique lies on a timeout: If the timeout expires the path must be discovery anew. If the network is very dense, i.e. if there are several paths between the source and destination, the probability of discovering a symmetric path is high, and eventually the protocol will discover it, as illustrated also in Fig. 3(b). Although this procedure is a simple protection against radio irregularities, it increases the data packet latency, which is proportional to the number of rounds used, and it increases the routing overhead in the network. It is worth noting that these problems are intermingled with the MAC dynamics [15]. The real impact of the radio randomness on the MAC layer depends on the type of access mechanism used. For example, in the case of IEEE 802.11, we can encounter two problems: the carrier sense problem, which is common to other CSMA protocols, and the handshaking problem, as shown in Fig. 4. In Fig. 4(a), node C does not hear the carrier from node $\mathrm{A}$ and a collision will happen at node $\mathrm{B}$. This is the well known hidden terminal problem of CSMA protocols. In IEEE 802.11, in order to solve this problem a handshake signalling 

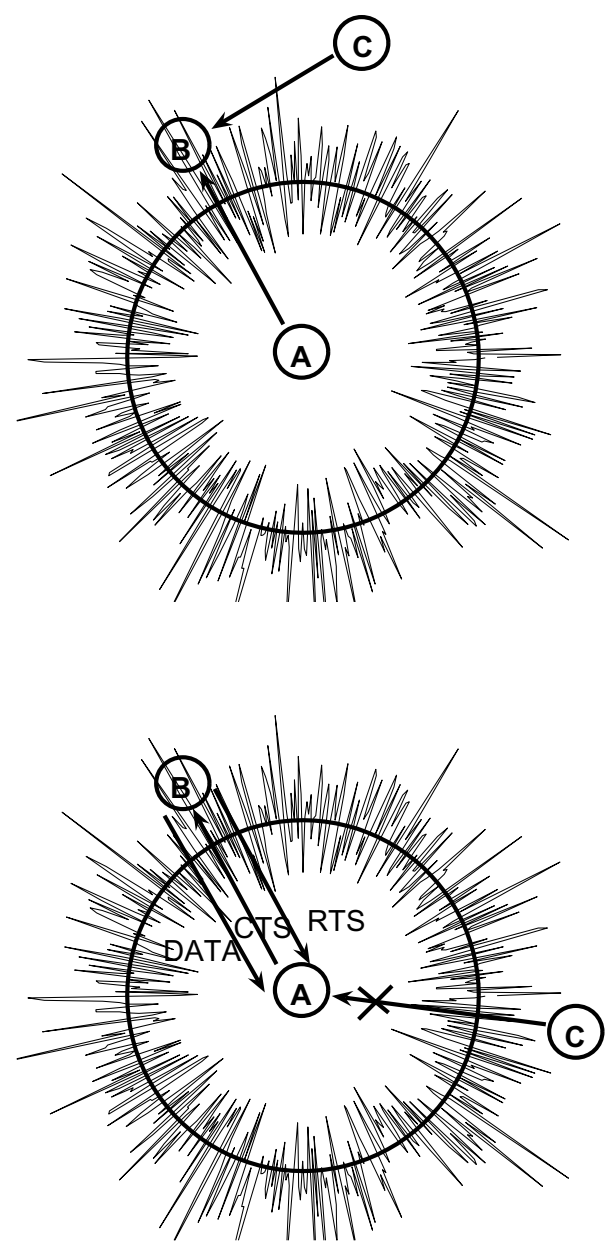

Fig. 4. Impact of radio irregularities on the MAC operations.

has been introduced. It uses two special signalling messages, for instance the Request To Send (RTS) and the Clear To Send (CTS) messages. Any node which hears the CTS message is supposed to wait long enough to allow the CTS's receiver node to send out data packets. However, radio irregularities can make incorrect also this solution, as shown in Fig. 4(b), where node C cannot hear the CTS message of node A. These effects are well analysed in [20], where the authors focused on the dependence between performance degradation of MAC/AODV protocols and the magnitude of the radio irregularity, measured by what they called Degree Of Irregularity (DOI). Although the WSN settings in [20] are specific of their test-bed, we believe that the same effects can be discovered in other scenarios.

\section{The impact on congestion control}

As the size of the WSN becomes large, some sort of congestion control is imperative in order to gather data from sensors in a way as reliable as possible. The congestion control directly affects the level of reliability of the detected event which the WSN is designed for. The concept of reliability in WSN depends on the application at hand. For example, if the network is used to track mobile objects, generally 
the user wants high precision of the object location. For other purposes, this precision can be lower, as in the case of ambient variables, for instance temperature or pressure. The precision can be quantified with a distortion measure, that is the average of the squared difference between the real value of the physical variable and the value reported at the monitoring node. The reliability depends also on other hardware parameters such as the signal quantization scheme of sensor nodes, the coding scheme of the transmitted data and the fault tolerance of electronic devices. In some cases, the reliability can require also bounds on the maximum detection latency, that is the maximum time during which the detected event is promptly provided at the monitoring node (or sink node). Nevertheless, in a WSN the detection of an event is a collaborative operation performed by more than one sensor node. Consequently, the bandwidth resource which is always a scarce resource should be adequately used among all sensor nodes.

It is well known that standard transport protocols as TCP do not fit the unique requirements of WSNs. In fact, TCP is based on a congestion window control: Every packet needs to be acknowledged by the receiver with an ACK packet. A mismatch on the sequence numbers of the transmitted packet and the acknowledged one means a congestion notification, usually due to overflow of buffers of forwarding nodes. Upon a loss, TCP reduces the congestion window, and it begins a re-transmission procedure. This scheme is clearly not optimal in WSN, where losses can be due to contention on the radio medium and radio irregularities as well. Here, we choose to focus on the data-centric model used in [2], where the end-to-end reliability is transformed into a bounded signal distortion concept. The resulting protocol has been called as Event-to-Sink Reliability Transport (ESRT). In this model, it is supposed that the WSN is composed of one sink node and several sensor nodes which report the reading of the physical event back to the sink node. The reporting frequency of sensors is defined as the number of packets sent out per unit of time. This frequency depends on several factors, as the quantisation step of sensors, the type of phenomenon, and the desired level of distortion perceived at the sink node. By exploiting the spatial and temporal correlation of the sensed data, the reporting frequency can be properly adjusted in order to meet the required distortion level. In our framework, we do not explicitly address the problem of a distortion, but we suppose a more general scheme. In the ESRT framework, the objective is selecting a subset of nodes around the event region and the best value of the reporting rate in order to achieve the distortion as low as possible. Low values of the reporting rate can lead to high distortion even with high nodes density, because of the packet losses. Here, we provide sensors also with a packet reliability scheme. That is, after sensing an event, every sensor node establishes a UDP connection towards the sink node, and it uses a repetition scheme for the packet transmission, i.e. it transmits multiple copies of the data packets reporting the details of the detected event. The setting of the reporting rate, $T_{r}$, depends on the sampling-encoding rate of the sensors, the type of correlation of the phenomenon and the nodes density. On the other hand, the control of this parameter affects the congestion and the mean latency of transmissions towards the sink node. The dependence of the congestion on the irregularities of the radio medium is the core of our contribution and is explained in the next section.

\section{Performance evaluation}

In this section, we present the simulation results about the impact of radio irregularities within a WSN. Firstly, we briefly describe the settings of our WSN. All simulations are performed by means of NS-2 simulator, with the support of NRL libraries. Since the number of scheduler events within a simulated WSN can be very high, we applied a patch against the scheduler module of NS-2 in order to speed up the simulation time [17]. The congestion control is carried out as explained in Section 5. We suppose also that the physical event is a discrete event, i.e. it emits a signal at constant rate (i.e. it is a monochrome signal). 
Table 1

Topology settings

\begin{tabular}{lc}
\hline \multicolumn{2}{c}{ GRID } \\
\hline step & $d=\frac{L}{\sqrt{N}-1}(\mathrm{~m})$ \\
service area size & $L^{2}=(800 \times 800)(\mathrm{m})^{2}$ \\
number of nodes & $N=16 \div 256$ \\
\hline
\end{tabular}

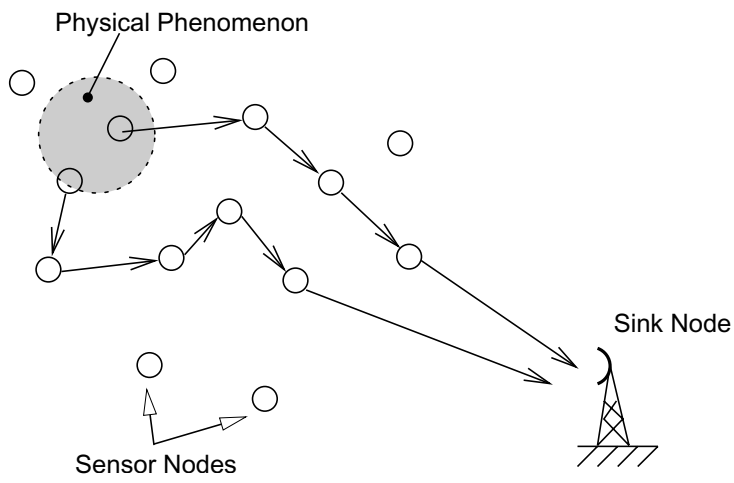

(a) Random topology

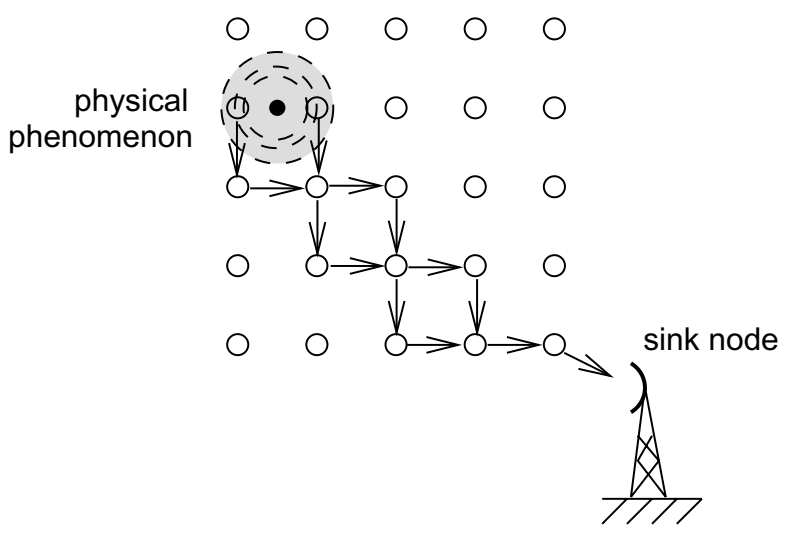

(b) Lattice topology

Fig. 5. Models of topology for the WSN.

\subsection{Topology}

For the physical layout of the WSN, two types of deployment has been studied so far: the random and the lattice deployments. In the former, nodes are supposed to be uniformly distributed inside the service area, while in the latter, nodes are vertexes of particular geometric shape, e.g. a square grid, as depicted in Fig. 5. In this case, in order to guarantee the connectedness of the network, we should set the transmission range of every node to the step size, $d$, which is the minimum distance between two rows (or columns) of the grid. In fact, by this way the number of links that every node can establish, a.k.a the node degree, is $D=4$. By using Cooper's theorem [6] along with some power control techniques, 
Table 2

Parameters of the radio model

\begin{tabular}{lc}
\hline RADIO MODEL & SHADOWING \\
\hline pathloss coefficient & $\beta=2.7$ \\
variance & $\sigma_{\mathrm{dB}}^{2}=16(\mathrm{~dB})$ \\
frequency & $916(\mathrm{MHz})$ \\
antenna & omni \\
\hline
\end{tabular}

Table 3

Settings of the sensor nodes

\begin{tabular}{lc}
\hline \multicolumn{2}{c}{ ENERGY PARAMETERS } \\
\hline initial energy & $E_{I}=7.5 J(=2.4 \cdot 3(\mathrm{mAhV}))$ \\
transmit power & $P_{T x}=0.660(\mathrm{~W})$ \\
receive power & $P_{R x}=0.395(\mathrm{~W})$ \\
sensing energy & $1 \mu(\mathrm{J})$ \\
\multicolumn{1}{c}{ OTHER PARAMETERS } \\
reporting rate & $T_{R}=0.1 \div 1(\mathrm{Kpps})^{1}$ \\
interface queue size & 50 (packets) \\
UDP packet size & 100 (bytes) \\
\hline
\end{tabular}

${ }^{1}$ Packet per seconds.

one could use also $D=2 .{ }^{4}$ However, we assume all nodes to be equal and then the degree is fixed to 4 . Nodes at the borders have $D=2$. The topology parameters are shown in Table 1 .

\subsection{MAC protocol}

Although not optimal for WSNs, we assume that the MAC protocol is the IEEE 802.11 standard. The receiver of every sensor node is supposed to receive correctly data bits if the received power exceeds the receiver threshold, $\gamma$. This threshold depends on the modulation scheme. ${ }^{5}$ As reference, we select parameters values according to the features of a commercial device (MICA2 OEM). In particular, for this device, we found that for a central frequency of $f=916^{\circ} \mathrm{MHz}$ and a data rate of $34 \mathrm{KBaud}$, we have a threshold (or receiver sensitivity) $\gamma=-98 \mathrm{dBm}$ [1]. The transmission range of sensor nodes should be at least $d$. However, in the case of a general radio model, we must take into account randomness of pathloss. Based on the grid step $d$ and on Eqs (1) and (3), we can set the maximum transmission range $\max \left(r_{0}\right)=d$. Accordingly, we can set the transmission power by solving $p=\operatorname{Prob}\left\{\left.P_{r}(d)\right|_{\mathrm{dB}}>\gamma\right\}=0.95$. By using the reasoning of Section 3, it is straightforward to show that:

$$
\left.P_{t}(d)\right|_{\mathrm{dB}}=\left[10 \beta \log _{10} \frac{d}{d_{0}}+\gamma-\operatorname{erfc}^{-1}(2 p) \sqrt{(2)} \sigma\right]++10 \log _{10}\left(\frac{d_{0}}{K}\right)^{2},
$$

where $\mathrm{erfc}^{-1}$ is the inverse of the standard error function. This formula provides the transmission power of each sensor, given a transmission range and a probability or rate of coverage $p$. This should not be confused with the sensing coverage of the WSN.

\footnotetext{
${ }^{4}$ By using the theorem in [6], we can say that a simple 2-regular network is almost surely strongly 2-connected.

${ }^{5}$ Other MAC factors affect the reception process, for example the Carrier Sensing Threshold (CST) and Capture Threshold (CP) of IEEE.802.11 used in NS-2.
} 


\subsection{Energy model}

The energy model concerns the dynamics of energy consumption of the sensor. A widely used model is as follows [9]. When the sensor transmits $k$ bits, the radio circuitry consumes an energy of $k P_{T x} T_{B}$, where $P_{T x}$ is the power required to transmit a bit which lasts $T_{B}$ seconds. By adding the radiated power $P_{t}(d)$, we have:

$$
E_{T x}(k, d)=k P_{T x} T_{B}+P_{t}(d) .
$$

Since packet reception consumes energy, by following the same reasoning, we have:

$$
E(k, d)=E_{T x}(k, d)+E_{R x}(k, d)=k P_{T x} T_{B}+P_{t}(d)+k P_{R x} T_{B},
$$

where $P_{R x}$ is the power required to correctly receive (demodulate and decode) one bit. In Tables 2 and 3 , we summarise the values of parameters used in our WSN. Let us note that the power values concern the power required to transmit and receive one bit, respectively. They do not refer to the radiated power at all. This is also the energy model implemented in the widely used NS-2 simulator.

\subsection{Simulations}

Based on the above settings, we use the following metrics. The goodput is defined at the sink node, and it is the received packet rate divided by the sent packets rate. Thus:

$$
G(\tau)=\frac{N_{r}(\tau)}{N_{s}(\tau)}
$$

where $N_{r}(\tau)$ is the number of received packets at the sink, and the $N_{s}(\tau)$ is the number of packets sent by sensor nodes which detected the phenomenon. These quantities are computed in a time interval of $\tau$ seconds. Since we are using an event-reliability approach for the congestion control, this metric can be considered as a measure of the perceived distortion at the monitoring node. The monitoring node can require a lower distortion by means of dedicated messages which instruct sensor nodes in how to adjust reporting rate or other sensing parameters. This adjustment will in turn affect the congestion level within the network. The entire system can be viewed as a control loop system with the required signal distortion as input and the sink node as comparison point. However, here we study the dependence of the congestion on the reporting rate only. The exact value of $\tau$ depends on the applications and the particular monitored event.

As long as the WSN is being used, a certain amount of energy will be consumed. The energy consumption rate directly affects the life-time of the network, i.e. the time after which the WSN is unusable. Accordingly, we define the energy consumed by the network in the detection interval $\tau$ as:

$$
\text { Energy consumed } \triangleq \frac{\text { Initial Tot. Energy }- \text { Final Tot. Energy }}{\tau} .
$$

However, in order to compare the performances of the scaled networks, it is better to define the mean energy depletion rate per node as:

$$
\Delta(\tau) \triangleq \frac{E_{I}-\bar{e}(\tau)}{\tau}=\frac{N E_{I}-\sum_{i=1}^{N} e_{i}(\tau)}{N \tau}
$$



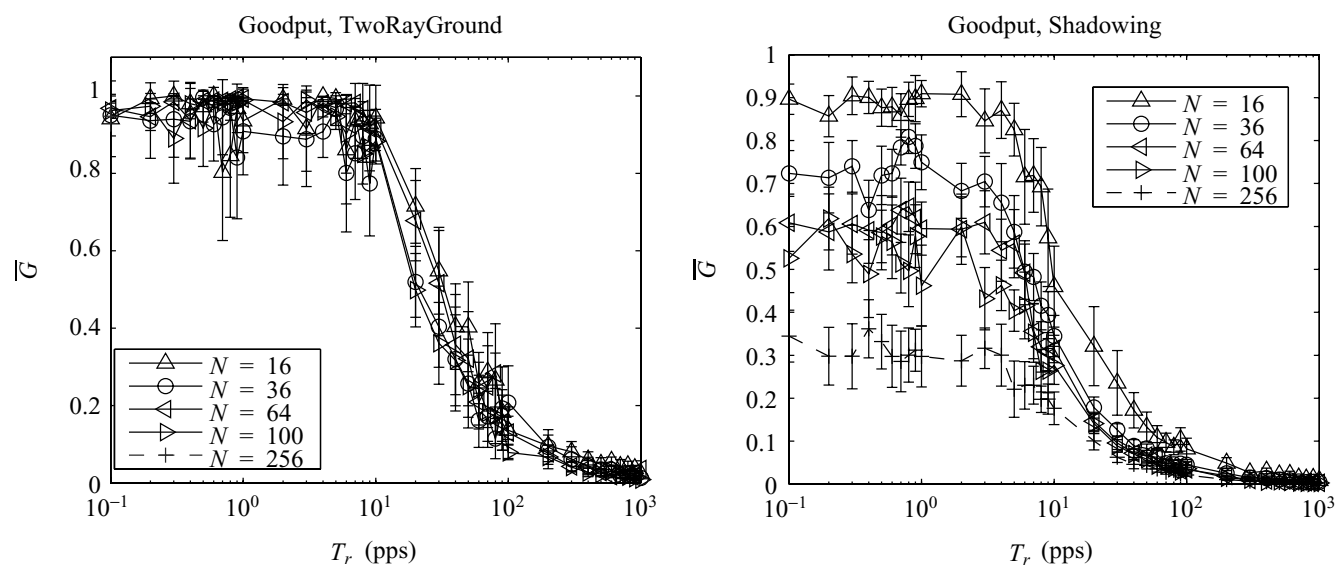

(a) AODV
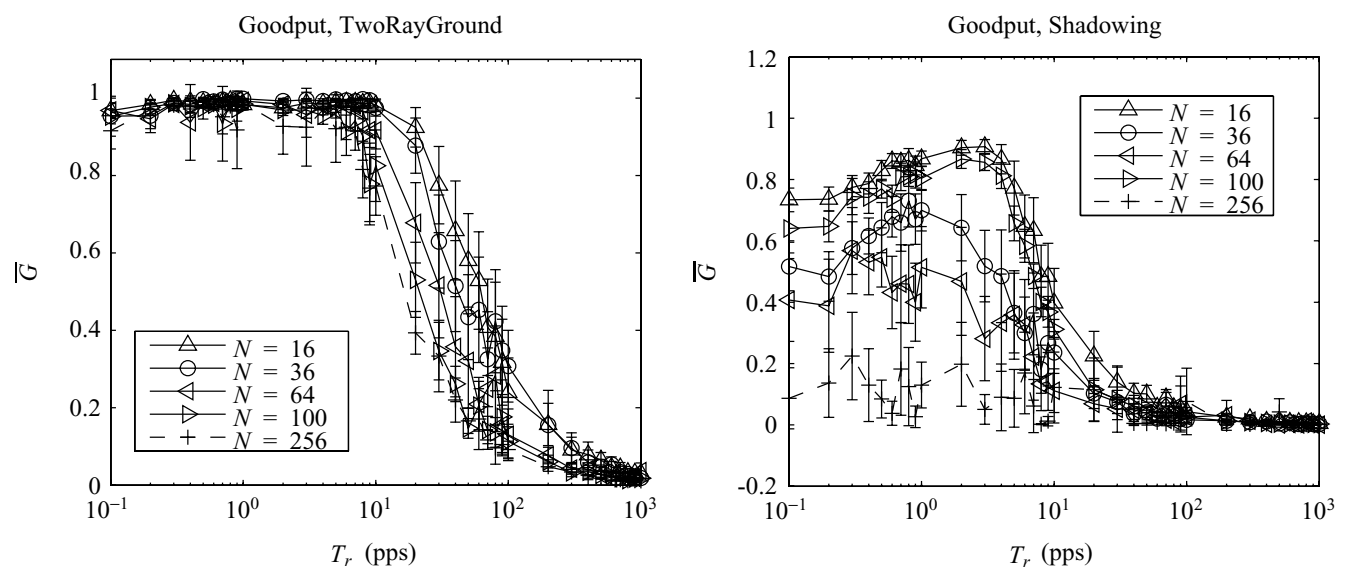

(b) DSR

Fig. 6. Sample averages of the goodput for two types of radio model.

where $e_{i}(t)$ is the node energy at time $t$ and the means are computed over the number of nodes. The number of nodes $N$ is set as power of integers in order to analyse the behaviour of the scaled versions of the network. The initial position of the phenomenon node is varied along the simulation runs. The sample averages of Eqs (6) and (7) are computed over 20 simulation runs and are plotted in Figs 6 and 7, respectively, with respect to the particular radio model used. We also show the $95 \%$ confidence interval of the sample averages. A first look tell us that the perceived goodput is a decreasing function of $T_{r}$. This was expected, because as $T_{r}$ increases, the capacity of the WSN limits the maximum number of packets per unit time which can be injected in. We can see this fact also by inspecting the number of received packet, as shown in Fig. 8.

We can clearly distinguish three operating zones. For low values $T_{r}$, the network is uncongested (just 30 data packets). From a particular value of $T_{r}$, the goodput drops abruptly. We have reached the network capacity. From this point on, increasing $T_{r}$ does not ameliorate the goodput and $N_{r}(\tau)$ is roughly constant. However, this does not happens for very small networks, e.g. for $N=16$.

Although these three zones are present regardless of the radio model, in the case of shadowing the goodput decreases with $N$. The explanation of this effect is not simple, because it is intermingled with the 

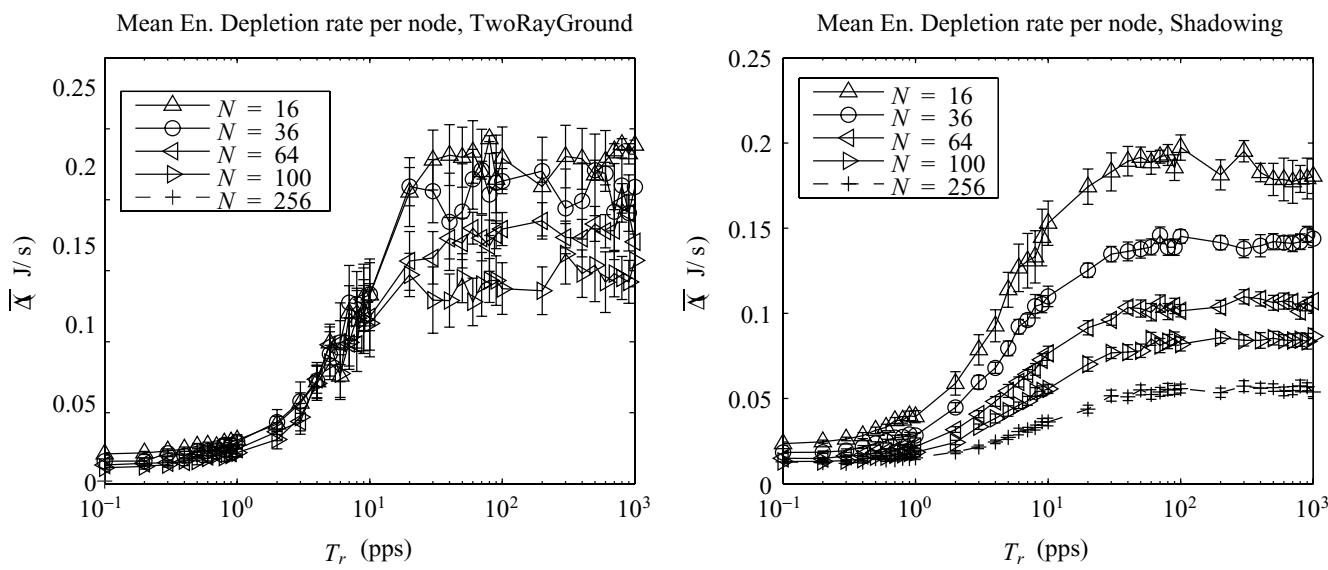

(a) AODV
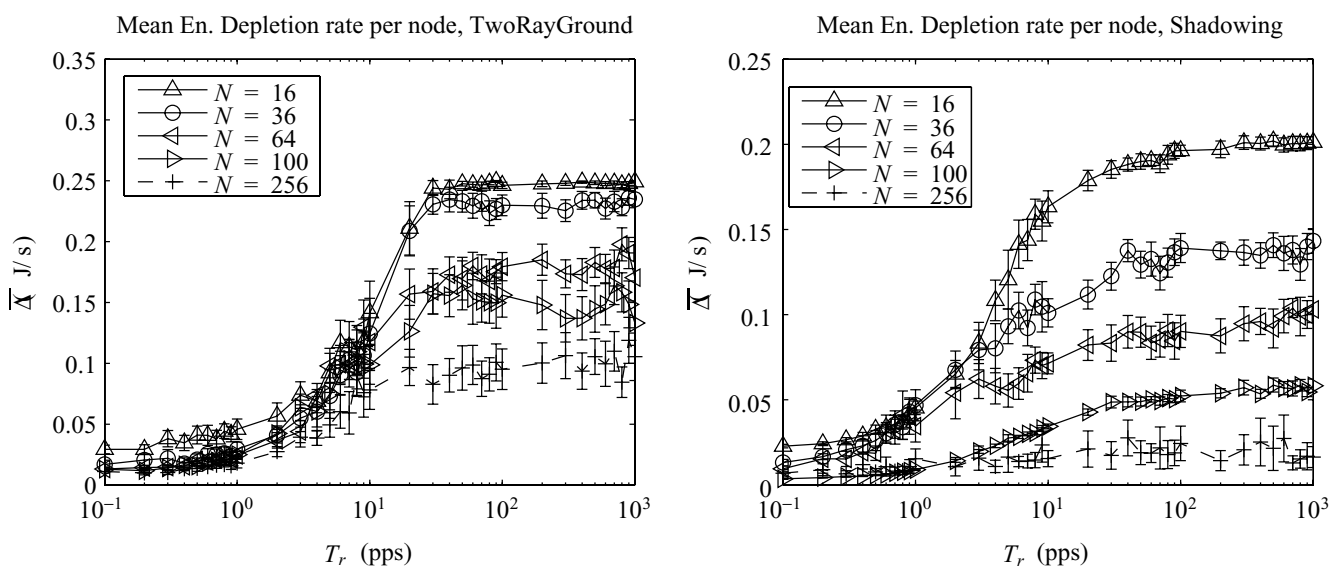

(b) DSR

Fig. 7. Sample averages of the energy depletion of the WSN with AODV and DSR in $\tau=30$ s.

dynamics of MAC and routing protocol. However, intuitively we can say that in the case of shadowing on demand routing protocols can discover paths which are to be re-discovered during the transmission of data packets. Thus, given a fixed detection interval, $N_{r}$ can be much lower than its value in the case of ideal radio model, where the discovered path does not change over time. ${ }^{6}$ It is well known that the shadowing destroys the regular topology of the lattice, by reducing the mean distance towards the sink node. However, for higher values of $N$, the augmented interfence level and the path instability seem to be predominant. Another aspect which affects the goodput is the network interface queue length of nodes which act also as routing nodes, although we did not analyse in this work. Note also that DSR performs worse than AODV, for low values of $T_{r}$ and $\tau$ (Fig. 6(b)). If all nodes had the same sensing range, the higher is the node density, the higher would be the number of nodes which can detect the phenomenon and the congestion level inside the network. The exact value of the sensing range depends on the desired coverage probability of the WSN, that is the probability that a point in the service area of

${ }^{6}$ This is true if we do not count the reliability of nodes, i.e. the probability of failure of sensor nodes. 

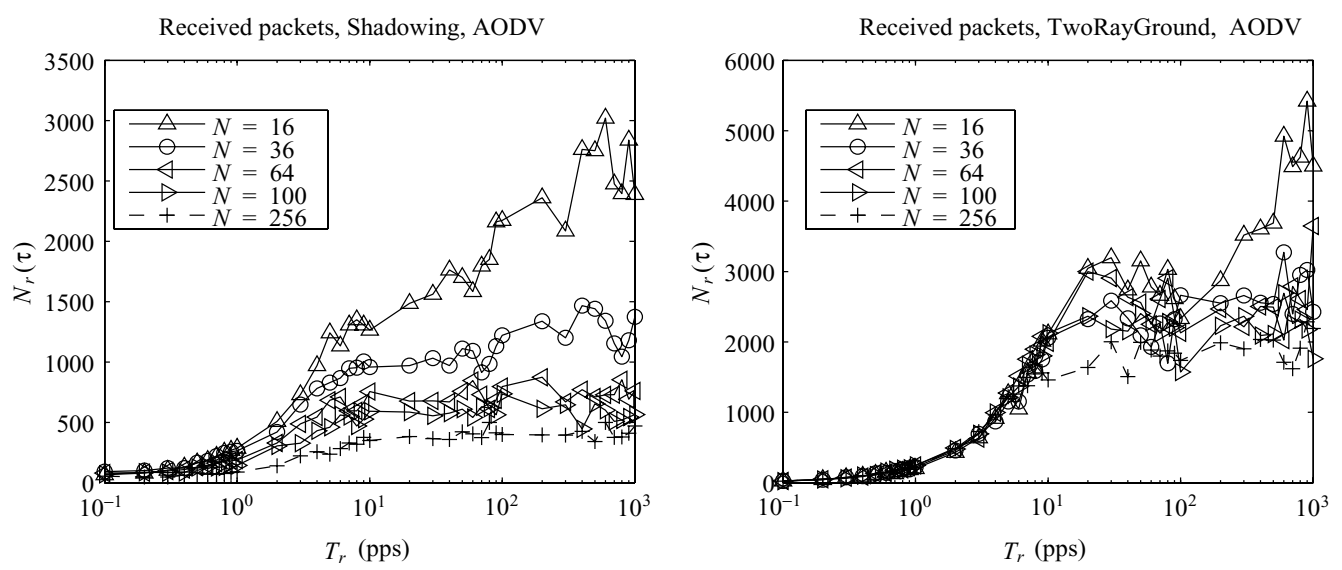

Fig. 8. Sample averages of the number of received packets at the sink node, AODV protocol.

the WSN is covered or "sensed" by at least one sensor node. There are analytical techniques to compute the minimum number of nodes to cover every point in a given area (see [18] and references therein), but all the available results are for ideal radio model only.

With respect to the energy depletion rate, we see from Fig. 7 that surprisingly in the shadowing case the WSN performs better than in the deterministic pathloss case. This result was expected because $\Delta(\tau)$ depends on the mean hop count of the network. If we measure the distance between a pair of nodes $(u, v)$ by the shortest path (if it exists) from $u$ to $v$, then the mean hop count in a network is the average of this distance over all possible pairs of nodes. If the network is random, the mean hop count must be averaged over "all possible" realisations of the network. As said before, the shadowing decreases the mean distance towards the sink node and then, in the average, a fewer number of nodes are interested in the data packet forwarding. Moreover, the performance of the lattice depends on the lattice step and the pathloss exponent. In fact, the energy loss can be formulated as follows. Given two configurations of the lattice with steps $d_{1}$ and $d_{2}$, respectively, it is straightforward to obtain the following relation between the consumed energies in a detection interval:

$$
\frac{E_{2}}{E_{1}} \sim\left(\frac{d_{2}}{d_{1}}\right)^{\alpha-2}
$$

where $E_{i}, i=1,2$ is the initial energy of nodes for the two network configurations, respectively. For $\alpha>2$ and $d_{2}>d_{1}$, the energy loss is greater than 1 and then, for a given coverage probability, small networks consume more than large ones, as shown in Fig. $7 .{ }^{7}$ Another explanation of this phenomenon is based on the theory of random graphs [3]. In this context, the network is modeled as a graph and the vertexes of the graph are nodes of the network. As said before, the parameter which affects the energy consumption is the hop count of the graph, that is the hop distance between two any nodes. In a pure random graph, where links are uncorrelated, the mean hop count is $\bar{h} \sim \frac{\ln N}{\ln D}$, while in a lattice with correlated links and $D=4$ as in our case, the mean hop count is $\bar{h} \sim O(\sqrt{N})$, which is greater than that of a pure random graph. However, in the case of shadowing, the behaviour of the graph tends to that of a random graph and then $\bar{h}$ decreases. The simulations reveal that for large scale networks the relative difference between deterministic and shadowing model can be as high as $50 \%$.

\footnotetext{
${ }^{7}$ This is the worst case, because we supposed a global flooding in a time unit.
} 


\section{Conclusions}

In this work, we faced the problem of radio irregularities in WSNs. Recently, this problem has been considered as a key factor in order to understand the real performance of algorithms and applications for multi-hop WSNs. As an example of the importance of this problem, we considered a WSN with a simple congestion control mechanism, built on the concept of event-reliability. To the best knowledge of the authors, only the work in [15] addressed the same problem, but in that work the effects of the irregularities of radio model have not been considered at all. We analysed the goodput and the energy depletion rate in a square lattice network. In general, in WSNs, the congestion problem is intermingled with the MAC and routing protocols behaviour. However, here we have shown how the goodput and the energy depletion rate change under the assumption of a general radio model. The importance of considering more realistic models is motivated also by the high variability (i.e. high confidence interval) we deliberately have shown in the simulation results. Thus, although widely used, few runs are not enough in order to get statistically meaningful values. These results can be easily extended to other routing and MAC protocols and other non-lattice topologies as well. Nevertheless, although difficult, an analytical approach to the presented results could help understanding and forecasting the behaviour of general networks.

\section{References}

[1] Crossbow technology, inc. http://www.xbow.com/.

[2] Özgür B. Akan and I.F. Akyildiz, Event-to-sink reliable transport in wireless sensor networks, IEEE/ACM Transactions on Networking 13(5) (2005), 1003-1016.

[3] B. Bollobas, Random Graph, Academic Press, 1985.

[4] A. Cerpa, J.L. Wong, L. Kuang, M. Potkonjak and D. Estrin, Statistical model of lossy links in wireless sensor networks, in ACM/IEEE Fourth International Conference on Information Processing in Sensor Networks (IPSN05), April 2005.

[5] T. Clausen and P. Jacquet, Optimized Link State Routing Protocol, IETF RFC 3626, 2003.

[6] C. Cooper, A note on the connectivity of 2-regular digraphs, Random Structures Algorithms 4 (1993), 469-472.

[7] I. Donward, Nrl's sensor network extension to ns-2. http://pf.itd.nrl.navy.mil/nrlsensorsim/, 2004.

[8] L. Girod, J. Elson, A. Cerpa, T. Stathopoulos, N. Ramanathan and D. Estrin, Emstar: a software environment for developing and deploying wireless sensor networks, In Proceedings of the 2004 USENIX Technical Conference, Boston, MA, 2004. USENIX.

[9] W.B. Heinzelman, A.P. Chandrakasan and H. Balakrishnan, An application-specific protocol architecture for wireless microsensor networks, IEEE Transactions on Wireless Communications 1(4) (October 2004), 660-670.

[10] ISI. Network simulator version 2. http://www.isi.edu/nsnam.

[11] P. Levis, N. Lee, M. Welsh and D. Culler, Tossim: accurate and scalable simulation of entire tinyos applications, In SenSys '03: Proceedings of the 1st international conference on Embedded networked sensor systems, New York, NY, USA, 2003. ACM Press, 126-137.

[12] W.M. Merrill, H.L.N. Liu, J. Leong, K. Sohrabi and G. Pottie, Quantifying short-range surface-to-surface communications links, IEEE Antennas and Propagation Magazine 46(3) (2004).

[13] C. Perkins, ed., Ad Hoc Networks, Addison-Wesley, 2001. Perkins Rappaport.

[14] T.S. Rappaport, Wireless Communications, Prentice Hall PTR, 2001.

[15] M.C. Vuran, V.C. Gungor and O.B. Akan, On the interdependency of congestion and contention in wireless sensor networks, in Proceedings of CST SenMetrics '05, July 2005.

[16] G.W. -Allen, K. Lorincz, O. Marcillo, J. Johnson and M. Ruiz an J. Lees, Deploying a wireless sensor network on an active volcano, IEEE Internet Computing 10(2) (Marhc 2006), 18-25.

[17] D.X. Wei, Speeding up ns-2 scheduler, http://netlab.caltech.edu/ weixl/technical/ns2patch, September 2005. California Institute of Technology.

[18] H. Zhang and J.C. Hou, is deterministic deployment worse than random deployment for wireless sensor networks? in: Proceedings of IEEE INFOCOM '06, April 2006, to appear.

[19] G. Zhou, T. He, S. Krishnamurthy and J.A. Stankovic, Impact of radio irregularity on wireless sensor networks, in: MobiSys '04: Proceedings of the 2nd international conference on Mobile systems, applications, and services, New York, NY, USA, 2004. ACM Press, pp. 125-138. 
[20] G. Zhou, T. He, S. Krishnamurthy and J.A. Stankovic, Models and solutions for radio irregularity in wireless sensor networks, to appear in ACM Transactions on Sensor Networks, 2006.

Tao Yang received BE from Hunan University, China in 2001. Presently, he is a Master Student at Graduate School of Engineering, Fukuoka Institute of Technology (FIT), Japan. His research interests include ad-hoc networks and sensor networks.

Giuseppe De Marco received the degree ("laurea") in Electrical Engineering in 1999 from University of Bologna, Italy. He received the PhD degree from University of Salerno, Italy, in 2004. From 2004 he is a Research Fellow at University of Salerno, and a Japan Society for the Promotion of Science (JSPS) Post Doctor Fellow Researcher at Fukuoka Institute of Technology, Japan. Dr. De Marco has been involved in the research activities of Coritel, a consortium sponsored by Ericsson Lab Italy, in the field of performance evaluation of signaling protocols. Its research interests are: network protocols, performance evaluation of wireless systems, inter-vehicle communication and sensor nets. He is member of IEEE.

Makoto Ikeda received BE from Department of Information and Communication Engineering, Fukuoka Institute of Technology (FIT), Japan in March 2005. He is a Master Student at Graduate School of Engineering, FIT. His research interests include high-speed networks, routing protocols, genetic algorithms and sensor networks. He is a Student Member of IPSJ.

Leonard Barolli received BE and PhD degrees from Tirana University and Yamagata University in 1989 and 1997, respectively. From April 1997 to March 1999, he was a JSPS Post Doctor Fellow Researcher at Department of Electrical and Information Engineering, Yamagata University. From April 1999 to March 2002, he worked as a Research Associate at the Department of Public Policy and Social Studies, Yamagata University. From April 2002 to March 2003, he was an Assistant Professor at Department of Computer Science, Saitama Institute of Technology (SIT). From April 2003 to March 2005, he was an Associate Professor and presently is a Full Professor, at Department of Information and Communication Engineering, Fukuoka Institute of Technology (FIT). Dr. Barolli has published more than 150 papers in referred Journals and International Conference proceedings. He was an Editor of the IPSJ Journal and has served as a Guest Editor for many International Journals. Dr. Barolli has been a PC Member of many International Conferences and was the PC Chair of IEEE AINA-2004 and IEEE ICPADS-2005. He was General Co-Chair of IEEE AINA-2006 and is Workshops Co-Chair of MoMM-2006 and IEEE AINA-2007. His research interests include network traffic control, fuzzy control, genetic algorithms, agent-based systems, ad-hoc networks and sensor networks. He is a member of SOFT, IPSJ, and IEEE. 

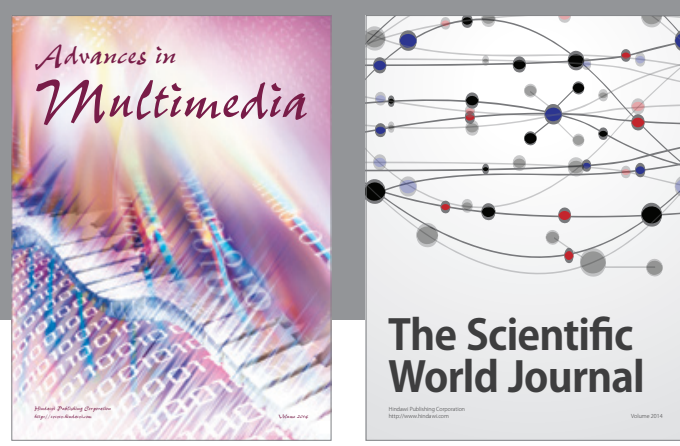

The Scientific World Journal
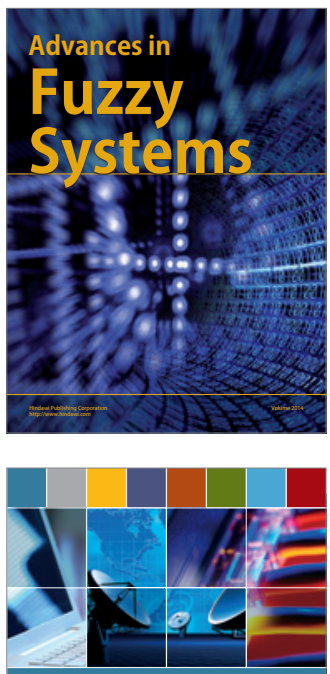

Computer Networks and Communications
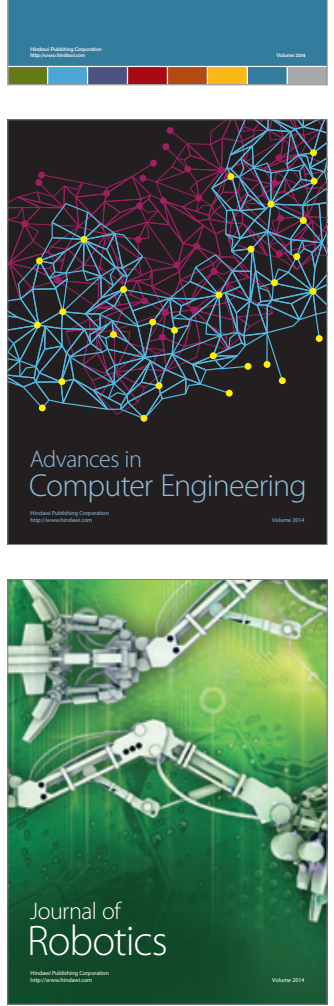
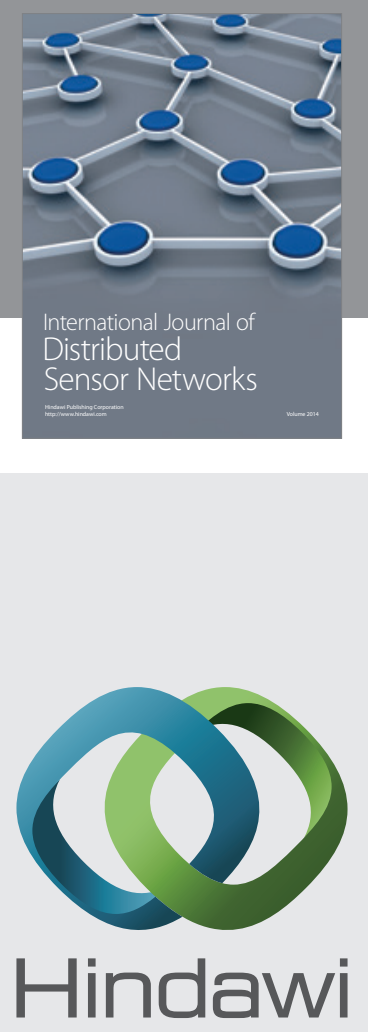

Submit your manuscripts at

http://www.hindawi.com
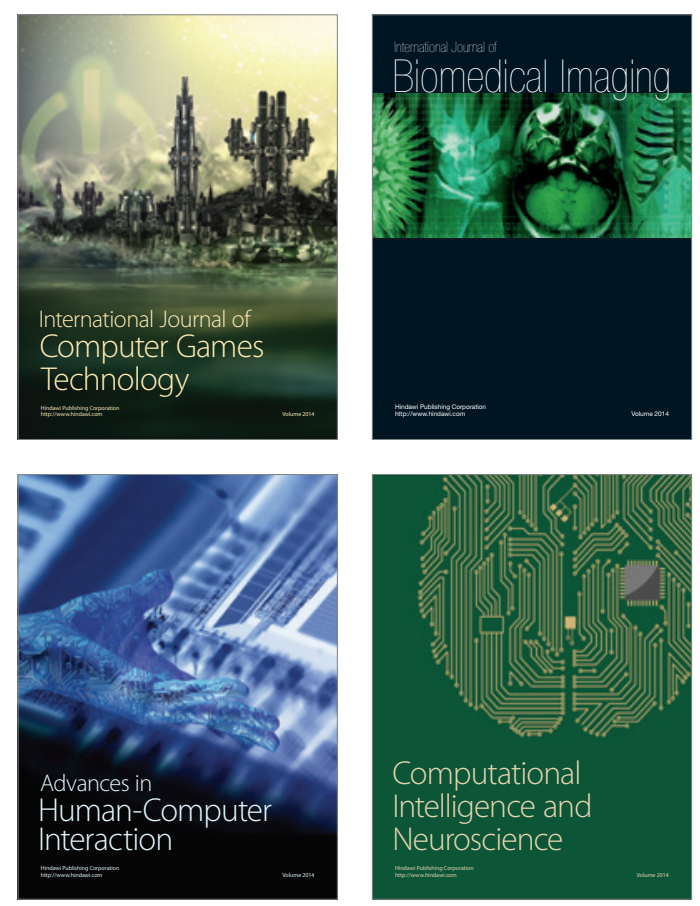
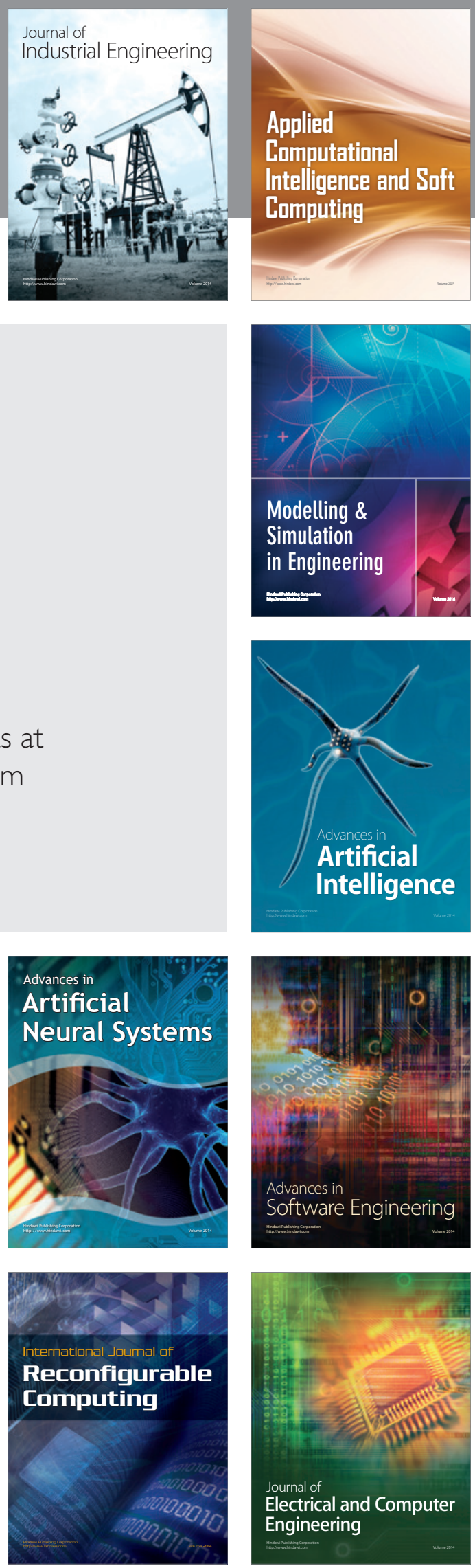